\title{
On the failure of distractor inhibition in the attentional blink
}

\author{
Paul E. DuX \\ Vanderbilt University, Nashville, Tennessee \\ AND \\ IRINA M. HARRIS \\ University of Sydney, Sydney, Australia
}

\begin{abstract}
We investigated whether a failure of distractor inhibition contributes to the magnitude of the attentional blink (AB). Subjects viewed dual-target rapid serial visual presentation (RSVP) streams, where the distractors that directly preceded and succeeded Target $2(\mathrm{~T} 2-1, \mathrm{~T} 2+1)$ were either identical to each other or different. Previously, Dux, Coltheart, and Harris (2006) found enhanced target report in RSVP due to repetition of distractors around Target 1, which was interpreted as evidence of distractor inhibition. Here, distractor repetition again attenuated the $\mathrm{AB}$, but only at lag 2 , the Target 2 position where $\mathrm{T} 2-1$ would have undergone attentive processing. Our results demonstrate that the distractor repetition effect is dependent on attention, and that a failure to inhibit distractors contributes to the $\mathrm{AB}$.
\end{abstract}

The limits of temporal attention are reflected by the attentional blink $(\mathrm{AB})$ : the reduced likelihood of reporting the second of two different targets in a rapid serial visual presentation (RSVP) stream if they are presented within 200-500 msec of one another (Raymond, Shapiro, \& Arnell, 1992). In a standard AB task, two targets are presented among a stream of distractor stimuli, and subjects are required to search for and report the targets. Thus, in such paradigms, capacity limitations associated with both the selection and consolidation of temporally distributed information are tapped.

Since its discovery, several theories of the AB have been proposed (Shapiro, Arnell, \& Raymond, 1997). One prominent account is that the $\mathrm{AB}$ occurs due to a bottleneck at the stage of consolidating information into working memory (Chun \& Potter, 1995). It has been hypothesized that the processing of RSVP stimuli proceeds through two distinct stages. In Stage 1, all of the stimuli in the stream are processed semantically; however, these representations are not durable and must undergo second-stage processing if they are to be available for report. This additional stage of processing is capacity limited and dependent on attention. Thus, during Target 1 (T1) consolidation, Target 2 (T2), if presented in close temporal proximity to the first target, must wait to receive limited capacity attentional resources. As a result, its volatile representation is more susceptible to decay and interruption from distractors.

The bottleneck model has been the dominant account of the $\mathrm{AB}$ over the last decade, but in recent times, a number of findings have led to the introduction of several new theories of the phenomenon. Di Lollo, Kawahara, Ghorashi, and Enns (2005; see also Olivers, van der Stigchel, \& Hulleman, 2007) found that when subjects were required to report three sequentially presented targets in an RSVP stream of digit distractors, performance did not differ on $\mathrm{T} 1$ and $\mathrm{T} 3$ (the equivalent to lag 2 in a standard $\mathrm{AB}$ experiment) when the targets were all letters. However, when T1 and T3 were letters and T2 was a digit, T3 accuracy was inferior to $\mathrm{T} 1$ accuracy - a result mirroring the standard $\mathrm{AB}$ effect. To account for these findings, Di Lollo et al. (2005) introduced the temporary loss of control hypothesis, which attributes the $\mathrm{AB}$ to the $\mathrm{T} 1+1$ (item directly following $\mathrm{T} 1$ ) distractor disrupting an input filter configured to the target set. In the case in which the three sequential targets are all letters, each target matches the filter, and consequently, limited-capacity processing resources can be devoted to encoding the targets. However, when T1 and T3 are letters and $\mathrm{T} 2$ is a digit, the second target does not match the input filter, which must then be reconfigured. This reconfiguration process draws on limited capacity resources, and consequently, fewer resources can be devoted to encoding later targets, leading to the $\mathrm{AB}$.

Nieuwenstein, Chun, van der Lubbe, and Hooge (2005; see also Nieuwenstein \& Potter, 2006) also suggest that the $A B$ occurs due to the inefficient selection of the second target in an RSVP stream. These researchers found that the $\mathrm{AB}$ was reduced when the second target was cued by a preceding distractor that shared target characteristics. This led to the formulation of the delayed-selection hypothesis of the $\mathrm{AB}$, which attributes the effect not to a consolidation bottleneck but to the failure to select the second target from the distractors at short lags due to 
limitations in processing resources. A final hypothesis is that the $\mathrm{AB}$ occurs due to the overinvestment of resources when processing the RSVP stream (Olivers \& Nieuwenhuis, 2006). Olivers and Nieuwenhuis demonstrated that $\mathrm{T} 1$ accuracy improved and the $\mathrm{AB}$ was attenuated when subjects performed an irrelevant task concurrently with the RSVP search. They hypothesized that performing the irrelevant task prevented subjects from inadvertently devoting too many resources to the distractors in the RSVP stream; consequently, at short lags, distractor interference was reduced and, as a result, the $\mathrm{AB}$ was attenuated.

All theories of the $\mathrm{AB}$ focus on accounting for why the second deficit occurs, and little emphasis is given to explaining how the system selects target stimuli from the distractors (see Loach \& Marí Beffa, 2003, for a study that does examine how targets are selected in RSVP). The importance of distractors in eliciting the $A B$ is demonstrated not only by the studies discussed above, but also by a range of other findings. First, even though distractors are not reported in RSVP, they nevertheless are processed semantically (Maki, Frigen, \& Paulson, 1997). Second, the mere presence of distractors (especially those immediately following the targets) is a crucial factor in causing the $\mathrm{AB}$; if these distractors are removed, the $\mathrm{AB}$ is substantially attenuated (Chun \& Potter, 1995; Giesbrecht \& Di Lollo, 1998). Finally, distractors interfere with targets both at featural and conceptual levels of processing (Dux $\&$ Coltheart, 2005). Thus, it is of considerable theoretical importance to elucidate the mechanisms involved in reducing distractor noise in RSVP.

Recently, Dux, Coltheart, and Harris (2006) have provided evidence that suggests that the inhibition of distractors that appear in close temporal proximity to $\mathrm{T} 1$ plays a key role in the selection of stimuli in RSVP. These researchers demonstrated that the $\mathrm{AB}$ was attenuated when the distractor items that directly preceded and followed $\mathrm{T} 1$ were identical to each other and drawn from a different category than were the targets (i.e., digits as opposed to letters). Dux et al. (2006) hypothesized that, under these conditions, the $\mathrm{T} 1+1$ item was inhibited at two levels: at a featural level, because feature detectors have brief refractory periods after initial activation, and at a conceptual level, due to the distractor set as a whole being inhibited, because it differed in category from the targets (Maki \& Padmanabhan, 1994). Consequently, when the distractor stimuli flanking the first target were identical and drawn from a different category than were the targets, the strength of $\mathrm{T} 1+1$ representation was reduced, causing an attenuation of its masking effectiveness. The outcome was more efficient $\mathrm{T} 1$ processing and a reduced blink.

Dux et al.'s (2006) results suggest that the successful selection of stimuli in RSVP is achieved by inhibiting those distractors that appear in close temporal proximity to the targets. However, does this mean that a failure of distractor inhibition directly contributes to the $\mathrm{AB}$ ? Put differently, is attention required to inhibit distractor stimuli in RSVP? If the distractor repetition effect (DRE) reported by Dux et al. (2006) is dependent on attentional resources being available, then it may be the case that, when targets appear within 200-500 msec of one another in an RSVP stream, subjects are unable to inhibit distractors that appear in close temporal proximity to the second target and, as a result, T2 is more susceptible to interference and, thus, more likely to be missed.

In order to test this hypothesis, we repeated the items that directly preceded and succeeded $\mathrm{T} 2(\mathrm{~T} 2-1$ and $\mathrm{T} 2+1)$ and manipulated the lag separating the two targets. If the DRE is dependent on attention, then distractor repetition should improve second target performance only when the distractor preceding T2 occurs outside the blink, because here it can be the subject of attentive processing; if this distractor falls within the blink, no attenuation of the $\mathrm{AB}$ is expected. On the other hand, if the DRE is not reliant on attentional resources, then the effect of distractor repetition should be uniform across lags. Therefore, the $\mathrm{T} 2$ temporal position of primary interest is lag 2 , because, in this condition, the distractor preceding T2 occurs directly after T1. Numerous studies have demonstrated that the item that appears in this position undergoes attentive processing along with the first target (Visser, Bischof, \& Di Lollo, 1999). Thus, if distractor inhibition is reliant on attentional resources being available, then a benefit of repeating the distractors should be observed only when the distractor preceding T2 appears directly after the first target.

\section{Experiments}

Two experiments are presented. Experiment 1 replicates Dux et al.'s (2006) original DRE methodology, by manipulating the similarity of the distractors that appear on either side of T1 in an RSVP stream. This experiment serves as a baseline with which the results of Experiment 2 are compared. In the second experiment, the similarity of the items flanking T2 is manipulated, the crucial result being the interaction between distractor repetition and lag.

\section{GENERAL METHOD}

\section{Subjects}

Thirty-five (11 male) undergraduate students at the University of Sydney participated in the study (15 in Experiment 1). All of the subjects reported normal or corrected-to-normal vision.

\section{Stimuli}

The stimuli were presented centrally in the same spatial location for $106 \mathrm{msec}$ each, with no interstimulus interval. Black letters and digits appeared on a white background. All of the stimuli were presented in Courier New font and subtended approximately $0.7^{\circ}$ visual angle at a viewing distance of $57 \mathrm{~cm}$. The experiment was programmed and conducted using DMDX software (Forster \& Forster, 2003) and was presented on a 19-in. flat CRT monitor with an $85-\mathrm{Hz}$ vertical refresh rate, controlled by a PC.

The targets were randomly selected from the alphabet, excluding the letters I, L, O, Q, U, and V, and were presented an equal number of times, with no pair of items appearing more than once. Targets were always presented in uppercase. Distractors were the digits 2-9, randomly ordered for each trial.

\section{Trial Structure}

All of the trials contained two black letter targets and eight black digit distractors, with a black " $\&$ " mask appearing at the end of each stream. T1 was presented in Serial Position 3, with T2 following an equal number of times at lags 2-6. In Experiment 1, in half the trials, the items directly before and after $\mathrm{T} 1(\mathrm{~T} 1-1$ and $\mathrm{T} 1+1)$ were identical (repeat trials), and in the other half they were different (nonrepeat 
trials). In Experiment 2, the distractors flanking T2 were either identical or different (see Figure 1).

\section{Procedure}

Each trial was initiated by the subject pressing the space bar. Trials began with a fixation cross for $300 \mathrm{msec}$, followed by the sequence of stimuli. Subjects were required to report the two letter targets at the end of each trial, when the prompt "Please recall the targets" appeared on the screen.

Subjects initially completed 20 practice trials that contained 2 trials of each condition. Experimental trials then commenced, with each subject completing two blocks of 50 trials. Five trials for each of the 10 conditions were presented in each block, in a random order.

\section{Design}

Both experiments employed a $2 \times 5$ repeated measures design. The independent variables were distractor repetition (repeat vs. nonrepeat) and $\operatorname{lag}(2,3,4,5$, and 6). The dependent variables were T1 and $\mathrm{T} 2$ given $\mathrm{T} 1$ correct (T2|T1) report accuracy.

\section{RESULTS}

\section{Experiment 1}

T1 accuracy. Repeating the distractors on either side of T1 facilitated report of the first target, accuracy being superior in repeat trials (95.9) in comparison with nonrepeat

\section{Experiment 1}
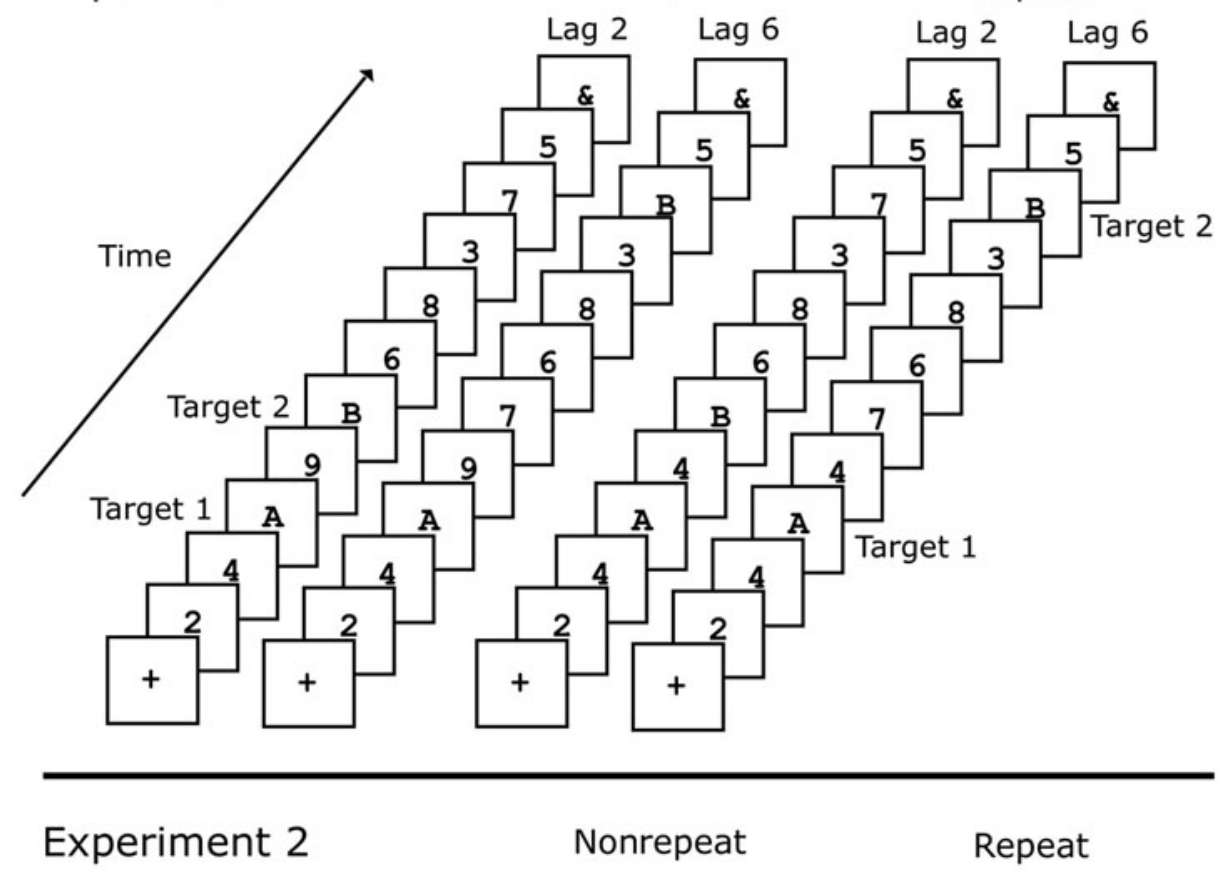

Nonrepeat

Repeat

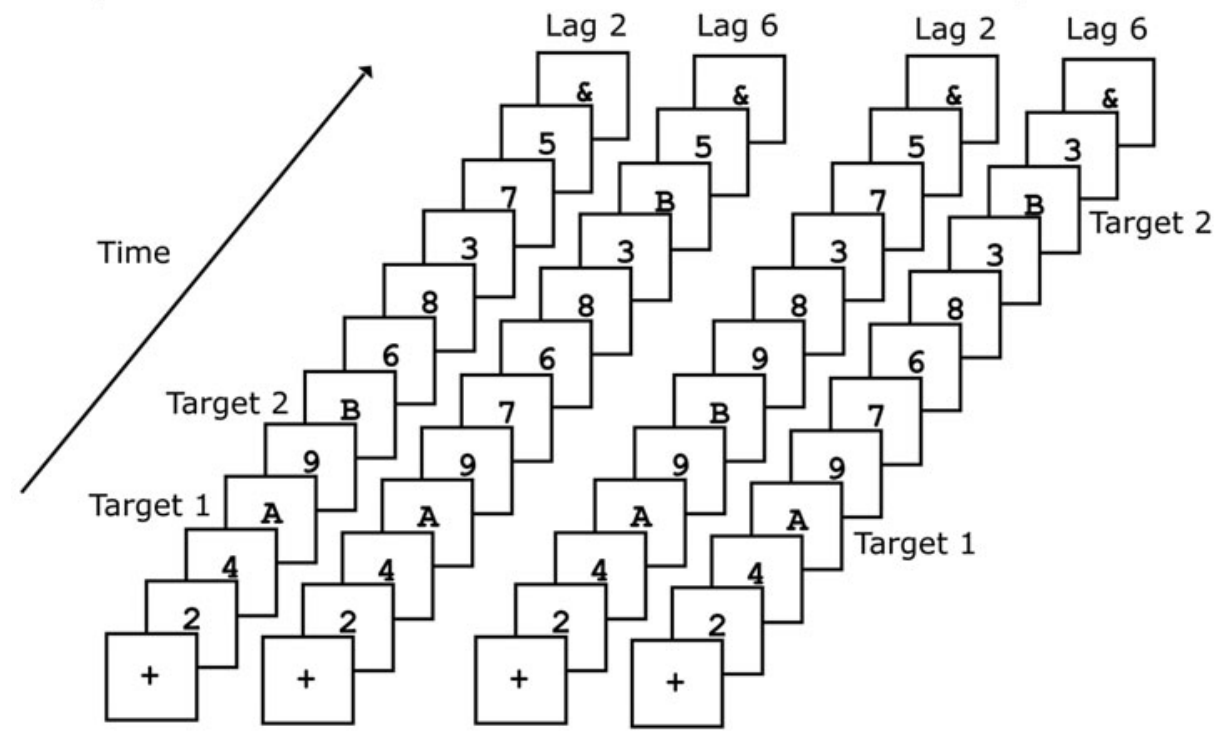

Figure 1. Example RSVP trials (nonrepeat and repeat, lag 2 and lag 6 conditions shown) from Experiments 1 and 2. 
trials $(92.7)[F(1,14)=7.5, p<.02]$. This finding suggests that the repeated distractor was a less effective mask for T1, and, as a result, the first target was easier to report.

T2|T1 accuracy. Figure 2 plots the mean T2|T1 accuracy in Experiment 1 as a function of distractor repetition and lag. As is evident from the graph, there was a significant $\mathrm{AB}$, with performance lower at early lags in comparison with later lags $[F(4,56)=23.8, p<.001]$. Importantly, there was a significant interaction between distractor repetition and lag $[F(4,56)=3.0, p<.03]$. This occurred because performance was superior for repeat distractor trials in comparison with nonrepeat trials at lag $2[t(14)=3, p<.01]$, but not at other lags. These results replicate those of Dux et al. (2006), demonstrating, once again, that repeating the distractors on either side of $\mathrm{T} 1$ reduces the masking strength of the $\mathrm{T} 1+1$ distractor, resulting in enhanced processing of the first target and a reduced $\mathrm{AB}$.

\section{Experiment 2}

T1 accuracy. There was no effect of distractor repetition on $\mathrm{T} 1$ accuracy in the second experiment, with neither the main effect of distractor repetition nor the interaction between distractor repetition and lag significant $\left(F_{\mathrm{s}}<\right.$ $2.1, p \mathrm{~s}>$.093). This finding makes sense when compared with the results of Experiment 1, given that, under the present conditions, the repetition of distractors would have only influenced the extent to which $\mathrm{T} 2$ was masked.

T2|T1 accuracy. Figure 3 plots the mean T2|T1 accuracy in Experiment 2 as a function of distractor repetition and lag. Examination of the graph reveals the existence of an $\mathrm{AB}$, with better performance at later lags in comparison with early lags $[F(4,76)=22.8, p<.001]$. Distractor repetition influenced overall performance, with accuracy superior in repeat trials (65.6) in comparison with nonre-

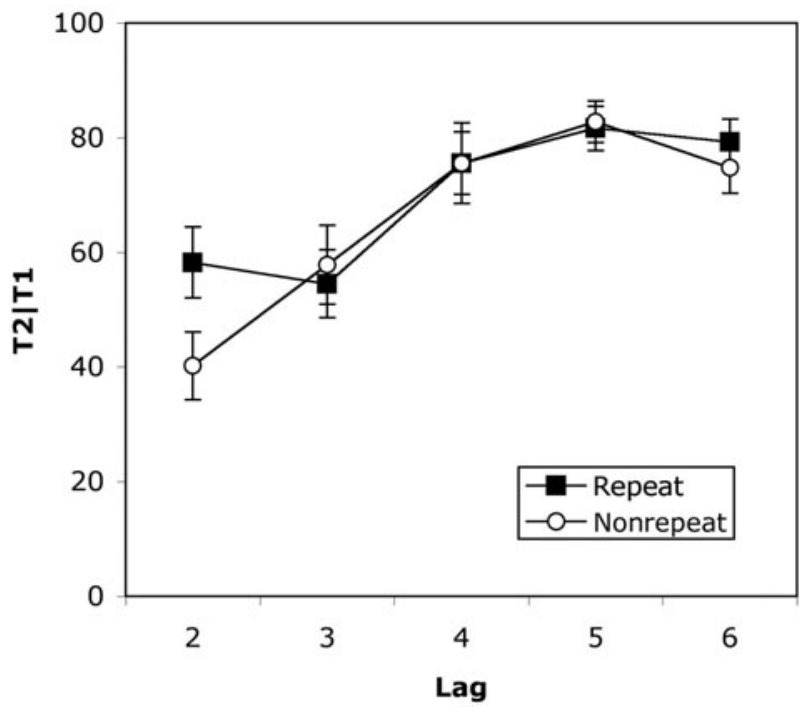

Figure 2. Mean percentage T2|T1 accuracy in Experiment 1 as a function of distractor repetition and lag. Error bars represent within-subjects standard error of the mean calculated separately for each lag. peat trials $(61.8)[F(1,19)=4.5, p<.05]$. Crucially, distractor repetition interacted with lag $[F(4,76)=3.8, p<$ $.001]$; accuracy differed significantly only between repeat and nonrepeat trials at lag $2[t(19)=3.4, p<.003]$, but not at other lags.

\section{DISCUSSION}

This study investigated whether a failure of distractor inhibition contributes to the AB. Experiment 1 demonstrated that presenting identical distractors directly before and after T1 not only enhanced first target processing, but also reduced the $\mathrm{AB}$. This finding is in line with previous research, which has demonstrated that, if the $\mathrm{T} 1+1$ distractor is replaced with a blank gap, T1 accuracy is enhanced and the AB attenuated (Chun \& Potter, 1995). We suggest that in Experiment 1, a repeated distractor was a less effective mask than a nonrepeated distractor because it had already been inhibited both at a featural and a categorical level (Dux et al., 2006). This caused its representation to be impoverished, and as a result it interfered less with $\mathrm{T} 1$ processing. In Experiment 2, repeating the distractors on either side of the second target also enhanced performance, which mirrors Giesbrecht and Di Lollo's (1998) finding that presenting a blank gap after the second target also reduces the AB. However, in this experiment, a benefit of repetition was observed only when the second target appeared at lag 2 . We suggest that a benefit of repetition was observed at lag 2 because in this condition, the $\mathrm{T} 2-1$ distractor was the subject of attentive processing, given that it was presented directly after the first target. As discussed above, numerous studies have demonstrated that the item that appears at this position (lag 1) undergoes attentive processing along with the first target (Visser et al., 1999). At the other lags, a benefit was not seen in the repeat condition because the T2-1 distractor appeared within the blink and therefore was not the subject of attention. From this, we conclude that the DRE occurs only if the first instance of a repeated distractor undergoes attentive processing. Put differently, distractors are only inhibited optimally if they occur outside the AB. This is consistent with the idea that a failure of distractor inhibition contributes to the second target deficit.

\section{Alternative Explanations of the DRE}

Although we have suggested that the DRE arises from inhibition both at the level of specific feature detectors and stimulus category (Dux et al., 2006; see also Maki \& Padmanabhan, 1994), it is important to consider alternative explanations of the effect. As discussed by Dux et al. (2006), the DRE is similar to another cognitive phenomenon, repetition blindness (RB), which is characterized by subjects' failure to report both occurrences of a repeated target in an RSVP stream if they appear within $500 \mathrm{msec}$ of one another (Kanwisher, 1987). RB is thought to occur as stimuli are encoded for overt report, which distinguishes it from the DRE, which occurs between identical stimuli that are not consciously encoded. Dux et al. (2006) noted that subjects, when questioned, never reported no- 


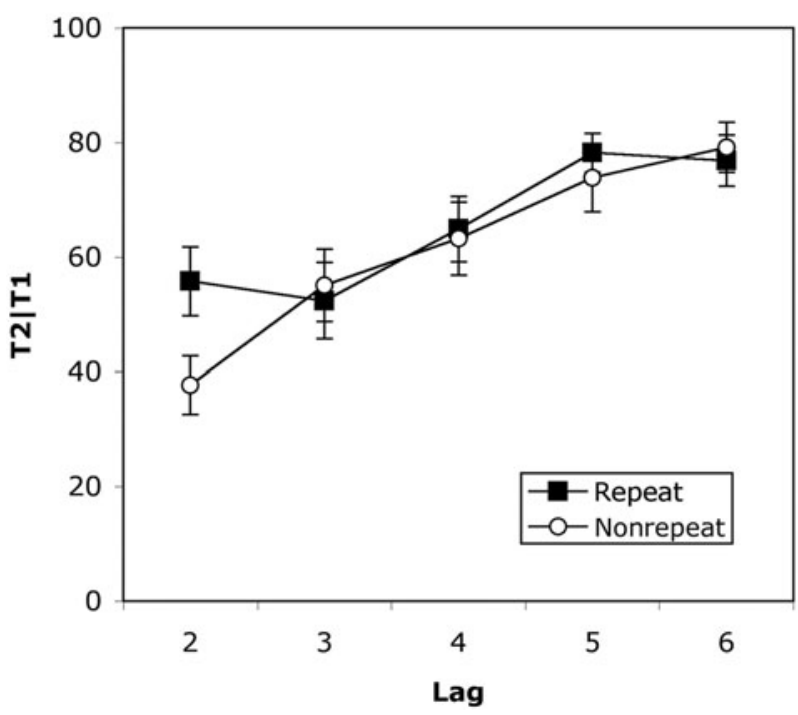

Figure 3. Mean percentage T2|T1 accuracy in Experiment 2 as a function of distractor repetition and lag. Error bars represent the within-subjects standard error of the mean calculated separately for each lag.

ticing the repeated distractors in the RSVP stream. A further difference between RB and the DRE is that, whereas $\mathrm{RB}$ can occur between different-case stimuli (e.g., $A$ and $a$ ), the DRE is dependent on the repeat items being identical. Thus, the mechanisms responsible for the two phenomena appear at least partially distinct. A potential problem for our inhibition account of the DRE is that a distractor in an RSVP stream can prime a later target with the same identity (Kanwisher, 1987). On the surface, this finding appears to be contrary to the predictions of the inhibition account. However, in this case, the distractor is drawn from the same category as the target and, as a result, the distractor set would not be inhibited and may, in fact, be enhanced, leading to priming between distractors and targets that share the same identity (Dux et al., 2006). Finally, another possible explanation for the DRE is that the repeat distractors on either side of a target were temporally grouped, which reduced the masking strength of the item following the target. To date, mechanisms of temporal grouping in vision (e.g., temporal integration; Coltheart, 1980) have been shown to operate only up to temporal durations of approximately $100 \mathrm{msec}$. In the present experiments, the onset of the first instance of the repeat distractor to the offset of the second was $300 \mathrm{msec}$. Thus, it appears that a temporal grouping account of the DRE is not viable.

\section{Implications for Theories of the AB}

As previously discussed, a number of new theories of the $\mathrm{AB}$ have been introduced, which hypothesize that a failure of target selection contributes to the AB (Di Lollo et al., 2005; Nieuwenstein et al., 2005; Nieuwenstein \& Potter, 2006; Olivers \& Nieuwenhuis, 2006; Olivers et al., 2007). The goal of the present study was to examine the mechanisms involved in target selection and to test whether they were dependent on attention. The results demonstrated that the DRE occurred only for stimuli that appeared outside the $\mathrm{AB}$ and thus were the subject of attentive processing. We propose that this reflects an inhibition of the distractors that appear in close proximity to targets in RSVP, and that this inhibition is modulated by top-down attention. Specifically, we propose that at short lags in a dual-target RSVP search, when limited-capacity attentional resources are devoted to $\mathrm{T} 1$ processing, fewer resources can be assigned to inhibiting the distractor set, thus causing the $\mathrm{AB}$.

We have hypothesized that attention is necessary for the DRE to occur; however, it is also possible that the inhibition of the T1+1 item in Experiment 2 may have occurred not because attentional resources were available at lag 1, but because this stimulus was so strongly attended by virtue of its proximity to $\mathrm{T} 1$ that it needed to also be strongly suppressed. This explanation fits well with the research of Di Lollo et al. (2005; see also Olivers et al., in press; Raymond et al., 1992), who provided evidence suggesting that the $\mathrm{AB}$ is caused by the extent to which attentional resources are devoted to processing the $\mathrm{T} 1+1$ item. Although this hypothesis can account for the results of the second experiment, the results of Experiment 1 are not as easily explained by this model. The T1 accuracy data in Experiment 1 suggest that it was the $\mathrm{T} 1-1$ item that was inhibited rather than the $\mathrm{T} 1+1$ stimulus, given that the first instance of the repeated distractor appeared directly before T1. Thus, we favor the hypothesis that the DRE was only present at lag 2 in the second experiment because distractor inhibition is dependent on attention, not because the $\mathrm{T} 1+1$ was so strongly attended that it needed suppression.

One interesting finding to note is that in Experiment 2, the DRE did not reappear at later lags, when the second target was no longer in the AB. At later lags, attention should again be available for inhibiting distractors and, therefore, it would be expected that distractor repetition would enhance T2 report. Thus, this null effect of distractor repetition at later lags is inconsistent with the hypothesis that the DRE is dependent on attention. A problem with assessing the DRE at lags 5 and 6 in Experiment 2 was that performance was close to asymptote; thus, the failure to observe a DRE at these lags is most likely due to a ceiling effect. Support for this hypothesis is that there was a significant DRE at lag 5 ( $p=.03$, one-tailed), when 6 subjects who demonstrated perfect performance (100\% accuracy) at this lag in the nonrepeat condition were excluded from the analysis. This finding strengthens our conclusion that attention is necessary for the DRE to be elicited.

\section{Conclusion}

The present study demonstrates that distractor inhibition plays an important role when selecting stimuli that are temporally distributed, and that a failure of this inhibition contributes to the AB. Future research should focus on further elucidating the mechanism(s) responsible for the DRE and characterizing not only the cognitive but also the neural fate of distractors (see Chelazzi, Miller, Duncan, \& Desimone, 1993). 


\section{AUTHOR NOTE}

This research was funded by Grant DP0557590 from the Australian Research Council, and I.M.H. was supported by an ARC Queen Elizabeth II Fellowship. We thank Bill Maki, Chris Olivers, and an anonymous reviewer for helpful comments on the manuscript and Claire Benito for assistance in testing subjects. Correspondence concerning this article should be addressed to P. E. Dux, Department of Psychology, Vanderbilt University, 428 Wilson Hall, 11121 st Avenue South, Nashville, TN 37203 (e-mail: paul.dux@vanderbilt.edu).

\section{REFERENCES}

Chelazzi, L., Miller, E. K., Duncan, J., \& Desimone, R. (1993). A neural basis for visual search in inferior temporal cortex. Nature, 363, 345-347.

CHUN, M. M., \& PotTer, M. C. (1995). A two-stage model for multiple target detection in rapid serial visual presentation. Journal of Experimental Psychology: Human Perception \& Performance, 21, 109-127.

Coltheart, M. (1980). Iconic memory and visible persistence. Perception \& Psychophysics, 27, 183-228.

Di Lollo, V., KaWahara, J., GhORAShi, S. M. S., \& EnNS, J. T. (2005). The attentional blink: Resource depletion or temporary loss of control? Psychological Research, 69, 191-200.

Dux, P. E., \& ColThearT, V. (2005). The meaning of the mask matters: Evidence of conceptual interference in the attentional blink. Psychological Science, 16, 775-779.

Dux, P. E., Coltheart, V., \& Harris. I. M. (2006). On the fate of distractor stimuli in rapid serial visual presentation. Cognition, 99, 355382.

Forster, K. I., \& Forster, J. C. (2003). DMDX: A Windows display program with millisecond accuracy. Behavior Research Methods, Instruments, \& Computers, 35, 116-124.

GIESBRECHT, B., \& Di LoLLO, V. (1998). Beyond the attentional blink: Visual masking by object substitution. Journal of Experimental Psychology: Human Perception \& Performance, 24, 1454-1466.

KANWISHER, N. G. (1987). Repetition blindness: Type recognition without token individuation. Cognition, 27, 117-143.
LoACH, D., \& Marí BefFa, P. (2003). Post-target inhibition: A temporal binding mechanism? Visual Cognition, 10, 513-526.

Maki, W. S., Frigen, K., \& PAUlson, K. (1997). Associative priming by targets and distractors during rapid serial visual presentation: Does word meaning survive the attentional blink? Journal of Experimental Psychology: Human Perception \& Performance, 23, 1014-1034.

MAKI, W. S., \& PadmanabHan, G. (1994). Transient suppression of processing during rapid serial visual presentation: Acquired distinctiveness of probes modulates the attentional blink. Psychonomic Bulletin \& Review, 1, 499-504.

NieUWenstein, M. R., ChUN, M. M., van Der Lubbe, R. H. J., \& Hooge, I. T. C. (2005). Delayed attentional engagement in the attentional blink. Journal of Experimental Psychology: Human Perception \& Performance, 31, 1463-1475.

NiEUWENSTEIN, M. R., \& PotTER, M. C. (2006). Temporal limits of selection and memory encoding: A comparison of whole versus partial report in rapid serial visual presentation. Psychological Science, 17, 471-475.

OLIVERS, C. N. L., \& NiEUWENHUIS, S. (2006). The beneficial effects of additional task load, positive affect, and instruction on the attentional blink. Journal of Experimental Psychology: Human Perception \& Performance, 32, 364-379.

Olivers, C. N. L., VAN der Stigchel, S., \& Hulleman, J. (2007). Spreading the sparing: Against a limited-capacity account of the attentional blink. Psychological Research, 71, 126-139.

Raymond, J. E., Shapiro, K. L., \& ARnell, K. M. (1992). Temporary suppression of visual processing in an RSVP task: An attentional blink? Journal of Experimental Psychology: Human Perception \& Performance, 18, 849-860.

ShAPIRO, K. L., ARnell, K. M., \& Raymond, J. E. (1997). The attentional blink. Trends in Cognitive Science, 1, 291-296.

VisSER, T. A. W., BISCHOF, W. F., \& Di LOLlO, V. (1999). Attentional switching in spatial and nonspatial domains: Evidence from the attentional blink. Psychological Bulletin, 125, 458-469.

(Manuscript received December 14, 2005; revision accepted for publication August 15, 2006.) 